\title{
Fully photonics-based radar demonstrator: concept and field trials
}

\author{
P. Ghelfi ${ }^{1}$, F. Laghezza ${ }^{1}$, F. Scotti ${ }^{1}$, G. Serafino ${ }^{2}$, S. Pinna ${ }^{2}$, D. Onori ${ }^{2}$, C. Porzi ${ }^{1}$, M. Scaffardi ${ }^{1}$, \\ A. Malacarne ${ }^{1}$, V. Vercesi ${ }^{2}$, E. Lazzeri ${ }^{1}$, A. Bogoni ${ }^{1}$ \\ ${ }^{1}$ CNIT, National Laboratory of Photonic Networks, via Moruzzi 1, 56124 Pisa, Italy \\ ${ }^{2}$ Scuola Superiore Sant'Anna, TeCIP Institute, via Moruzzi 1, 56124 Pisa, Italy \\ paolo.ghelfi@cnit.it
}

\begin{abstract}
This work shows the concept, performance, and field-trials of the first photonics-based radar. The comparative in-field experiments in aerial and naval scenarios against a state-of-the-art commercial system show the photonics potentials in enabling software-defined radars. OCIS codes: (060.5625) Radio frequency photonics; (280.5600) Radar; (140.4050) Mode-locked lasers
\end{abstract}

\section{Introduction}

The requirements of resolution, sensitivity, and flexibility of future multifunctional radar systems are pushing the development of reconfigurable and software-defined-radio (SDR) radar transceivers, capable of managing wideband waveforms over high-frequency carriers with the phase stability required by coherent pulse-Doppler processing. Thus, the evolution of future radars strongly depends on the progress of electronic digital components as the high speed direct digital synthesizers (DDSs) and the analog-to-digital converters (ADCs). So, bandwidth and precision of digital electronics represent the limit of current radar system performance. On the other hand, microwave photonics technologies promise to match tomorrow's system needs thanks to its inherent high frequencies and ultra-wide bandwidths [1]. Following this approach, the ERC-funded PHODIR project [2] has aimed at designing and implementing the architecture of a radio-frequency transceiver for fully digital radar systems, entirely based on photonics [3]. In this work, we review the architecture and the characterization of the PHODIR system, and we report on the latest field-trial demonstrations. These in-field experiments have assessed the performance and functionality of the developed radar demonstrator, through the detection of non-cooperating aerial and naval traffic and through the direct comparison with a state-of-the-art commercial solid-state coherent radar. The reported experimental results confirm the excellent potentials of the photonics approach of the PHODIR demonstrator, highlighting its high performance and unprecedented flexibility. Finally, we comment on the possible future developments of photonics-based radars, from both the technological and architectural viewpoints.

\section{Performance of the photonics-based radar transceiver}

The architecture of the PHODIR system is reported in Fig. 1 (left) [4]. The photonic generation of high-quality, flexible, and high-frequency RF signals exploits the heterodyne detection of modes from a mode-locked laser (MLL). The radar waveform is generated at a low intermediate frequency (IF) by a DDS, and modulates the MLL signal in an electro-optical modulator. The optical signal (composed of original MLL modes and new sidebands) is then detected by a photodiode (PD), and a radio-frequency (RF) filter selects the desired beating frequency without resorting to electrical mixers. Hence, the carrier frequency can be arbitrarily chosen as an integer multiple of the pulses repetition rate, plus the digitally generated and highly stable IF that holds the radar waveform and guarantees a continuous tunability. The filtered RF signal is then sent to a high-power amplifier to be transmitted from the antenna. Once the radar signal has been back-scattered from a target, it is received by the antenna, amplified, and filtered. Then the received RF waveform is used to modulate the signal from the MLL (optical sampling), generating replicas of the detected signal spectrum as sidebands of the MLL modes. Finally, a PD detects the optical signals and performs the down-conversion of the received echo from the RF frequency back to the original IF, again without electrical mixers. An ADC eventually digitizes the down-converted signal at IF. The proposed architecture therefore exploits a single MLL for both the radar transmitter and receiver. Besides the radar pulses, the photonic transmitter also generates a continuous-wave reference signal which is used to implement a coherent radar deriving the targets' speed.

The photonics-based transceiver (without an RF front-end) has been characterized in a laboratory environment, and compared with the performance of state-of-the-art electronic radar transceivers [4]. The advantages of the photonic approach are evident in the extreme flexibility in the generated carrier frequency ranging up to $40 \mathrm{GHz}$ (while electronic radars have fixed frequency), in the arbitrary modulation capability, and in the precision of the digitization for any input frequency (effective number of bits (ENOB) $>7$ up to $40 \mathrm{GHz}$, while electronic transceivers guarantee a direct digitization with $\mathrm{ENOB}<8$ only up to $2 \mathrm{GHz}$ ). These are fundamental features for enabling the SDR paradigm in future radars. 


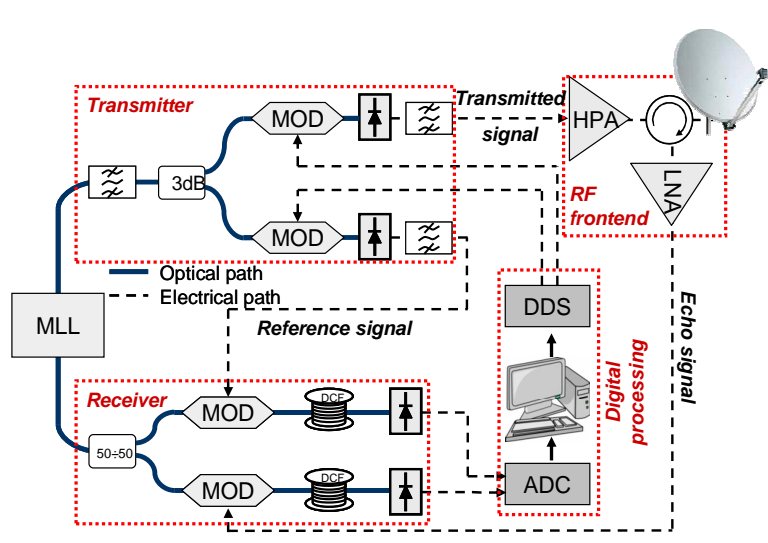

\begin{tabular}{|l|l|l|}
\hline \multicolumn{1}{|c|}{ Parameters } & \multicolumn{1}{c|}{ PHODIR } & \multicolumn{1}{c|}{ SEAEAGLE } \\
\hline Peak Power & $50 \mathrm{~W}$ (@ WR90) & $100 \mathrm{~W}$ (@ WR90) \\
\hline RF frequency (MHz) & $9880 \div 9920$ (continuous) & $9300 \div 9920$ (step) \\
\hline Max bandwidth & $40 \mathrm{MHz}$ & $40 \mathrm{MHz}$ \\
\hline Noise figure & $8 \mathrm{~dB}$ & $5 \mathrm{~dB}$ \\
\hline MDS & $-87 \mathrm{dBm} @ 40 \mathrm{MHz} \mathrm{BW}$ & $-90 \mathrm{dBm} @ 40 \mathrm{MHz} \mathrm{BW}$ \\
\hline Max processing gain & $31 \mathrm{~dB}$ & $34 \mathrm{~dB}$ \\
\hline Frequency accuracy & $120 \mathrm{ppm}(13 \mathrm{fsec})$ & $100 \mathrm{ppm}(10 \mathrm{fsec})$ \\
\hline Max range & $18 \mathrm{NM} \mathrm{(cargo} \mathrm{target)}$ & $24 \mathrm{NM} \mathrm{(cargo} \mathrm{target)}$ \\
\hline Pulsewidth & $0.2 \div 10 \mu \mathrm{sec}$ & $50 \mathrm{nsec} \div 26 \mu \mathrm{sec}$ \\
\hline PRF & $1 \div 12 \mathrm{KHz}$ & $661 \div 1112 \mathrm{~Hz}$ \\
\hline Modulation format & Any & Chirp \\
\hline
\end{tabular}

Fig. 1. Left: PHODIR transceiver architecture. Right: Specifications of the PHODIR demonstrator, compared with the SEAEAGLE by GEM elettronica.

\section{The radar demonstrator and the field trials}

To validate the PHODIR transceiver in a realistic surveillance operation, a radar demonstrator has been implemented, adding to the photonics-based transceiver an RF front-end (RF circulator, switches, amplifiers, filters, and monostatic antenna) for a signal carrier at $9,900 \mathrm{MHz}$, with a maximum instantaneous bandwidth of $40 \mathrm{MHz}$ due to the bandwidth of the exploited RF filters. The main specs of the demonstrator are reported in Fig. 1 (right). This demonstrator has been tested in several field trials.

The first field trial has been run from the lab roof, pointing at the air traffic from the close airport of Pisa, Italy [4]. The system was set to transmit a standard pulse train with $1 \mu$ s pulsewidth (PW) and $10 \mathrm{kHz}$ pulse repetition frequency (PRF), for a range resolution of $150 \mathrm{~m}$ over $15 \mathrm{~km}$ of unambiguous distance. The coherent integration time (CIT) was $20 \mathrm{~ms}$, corresponding to about 200 integrated pulses for a Doppler resolution of $0.55 \mathrm{~m} / \mathrm{s}(2 \mathrm{~km} / \mathrm{h})$ over an unambiguous velocity of $76.4 \mathrm{~m} / \mathrm{s}(275 \mathrm{~km} / \mathrm{h})$. The transmitted peak power was set to $20 \mathrm{~W}$. These features have allowed the correct detection of civilian airplanes during the take-off/landing maneuvers.

A second trial has been run in a maritime scenario at the port of Livorno, Italy, giving the opportunity to exploit the full-digital capabilities of the demonstrator [5]. The settings of the radar have been changed to fit two different case studies. The first one aimed at a long-range detection, targeting the cargo ships and ferries offshore. The radar pulse was modulated with a 13-bit Barker code allowing a range resolution of about $11 \mathrm{~m}$, and the CIT was extended to $100 \mathrm{~ms}$ for a Doppler resolution of $0.15 \mathrm{~m} / \mathrm{s}$. This way it has been possible to detect the slow speed as well as the shape of the targeted ships. Then the system was set for the short range detection of maneuvering targets into the harbor area. The PW was shortened to 400ns and compressed with a 13-bit barker code, for a range resolution of about $5 \mathrm{~m}$. These measures have allowed to clearly detecting even small boats moving within the harbor. It is important to note that these maritime measurements have not registered any spreading of the detected Doppler speed, usually present due to the sea clutter, in accordance with the high stability of the generated signal.

Recently another maritime field trial has been run in cooperation with GEM elettronica in the area around the port of San Benedetto del Tronto, Italy, where the detections obtained by the PHODIR radar system have been compared to the ones by a commercial coherent radar in the X band, the SEAEAGLE, the top-product of GEM elettronica [6]. A preliminary lab comparison (see Fig. 2 right) has highlighted very similar fundamental features for the two systems: the minimum detectable signal (MDS) has turned out to be $-90 \mathrm{dBm}$ for the SEAEAGLE and $87 \mathrm{dBm}$ for the PHODIR, while the noise figure has been measured to be respectively $5 \mathrm{~dB}$ and $8 \mathrm{~dB}$. These must be considered as promising results, being the PHODIR system at a demonstrator stage only, realized with standard, non-dedicated photonic devices. Moreover, the photonics-based radar has shown a superior flexibility (PW, PRF, pulse modulation type) and bandwidth. Then the two systems have been directly compared in a field trial, acquiring the same maritime environment. The two radars have been set to transmit a linear chirp with a frequency deviation of $10 \mathrm{MHz}$ over a PW of $1 \mu \mathrm{sec}$ and a PRF of $10 \mathrm{kHz}$, with a transmitted peak power of about $50 \mathrm{~W}$. The CIT has been set to $7.5 \mathrm{~ms}$, corresponding to the rotation of $1^{\circ}$ of the two similar rotating antennas exploited in the trials. The resulting resolution has been $15 \mathrm{~m}$ and $2 \mathrm{~m} / \mathrm{s}$. Both the systems have scanned the area around the port. Fig. 2 (A) shows the PHODIR detection trace overlaid to the satellite map of the port: the coastal area and the harbor shape are well represented, including the breakwater lines and a small boat at about 0.42 nautical miles (NM) with a detected radial velocity of 5 knots $(2.57 \mathrm{~m} / \mathrm{s})$. Fig. 2 (B) and (C) report the plan position indicator (PPI) plots for the same scene taken by the PHODIR and SEAEAGLE radars, respectively. While the PHODIR system under the current 
settings can analyze a range of $8 \mathrm{NM}$, due to its fixed settings the SEAEAGLE can analyze a range of $6 \mathrm{NM}$ only. Therefore, while the PHODIR has detected 5 targets, the SEAEAGLE has reached 4 of them. Besides this, the plots show a perfect matching in the visualized scenario.
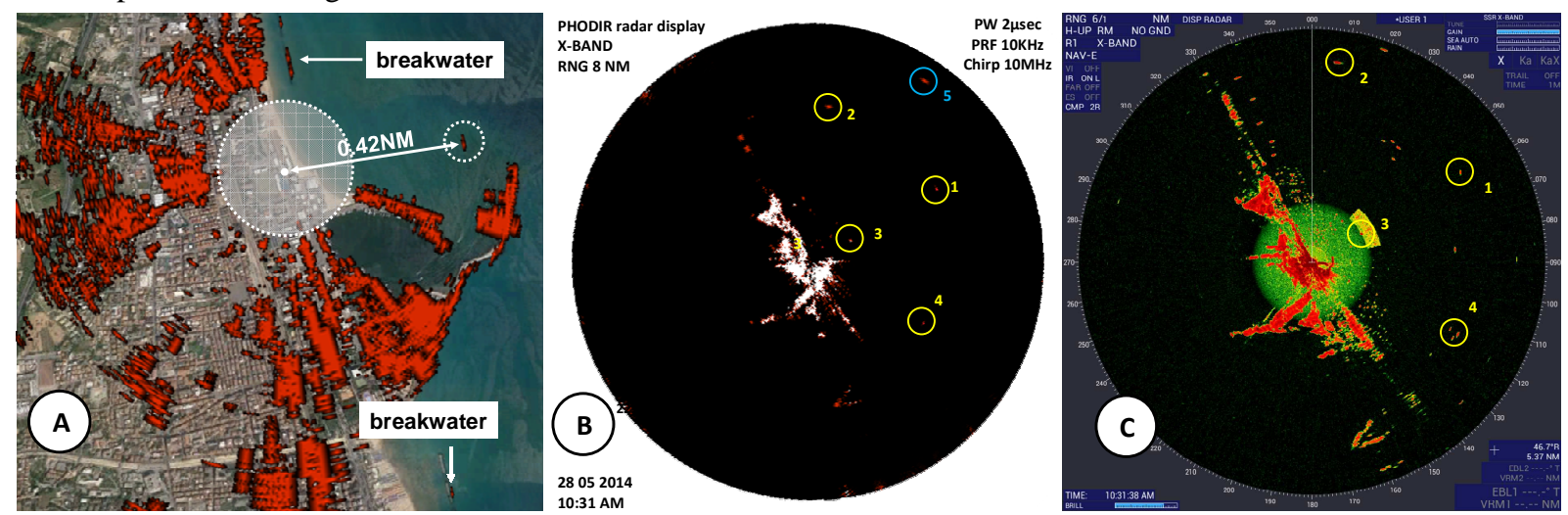

Fig. 2. (A) PHODIR radar detection of the area around the port of San Benedetto del Tronto, Italy. (B),(C) Comparison of the plan position indicator plots from the PHODIR and SEAEAGLE systems respectively, acquiring the same scene.

\section{Future developments}

The reported characterizations have demonstrated the unprecedented flexibility of the photonics-based radar transceiver, which allows enabling the SDR paradigm. Besides this, the results from the field trial experiments have assessed the good performance ensured by the radar demonstrator, which has turned out to be comparable with state-of-the-art commercial systems.

Several architectural developments can be imagined starting from the PHODIR scheme, further exploiting the potentials of photonics. For instance, we have already proposed to exploit the wide available optical spectrum to implement a photonic beamforming network feeding a phased arrayed antenna [7]. Moreover, the photonic core of the system could be doubled to implement a synchronous dual-frequency radar exploiting a single high-stability laser source [8]. Another possibility enabled by photonics is the implementation of a hybrid radar/lidar system, with peculiar synchronous and multispectral capabilities.

Even better performance is expected from the implementation of the PHODIR architectures through integrated photonic techniques. In fact, the specific design of photonic components as tunable narrowband filters can boost the system flexibility much further (for instance, avoiding the need for fixed RF filters). Moreover, an integrated realization is also expected to reduce the size and weight of the photonics-based radar transceiver, and to increase its stability (lower sensitivity to thermal fluctuations and vibrations).

Finally, the developments of the photonics-based radar transceiver can positively affect the RF transceivers at large, i.e. not confined to the radar application [9]. The photonic approach is in fact expected to allow the establishment of a new paradigm of RF systems, with improved performance, unprecedented flexibility, and reduced size and weight.

The authors acknowledge the port authorities of Livorno, and Dr. V. Malaspina and Dr. L. Banchi at GEM elettronica. This work has been supported by the EU projects PHODIR, PETRA, ROAM, FiWin5G, and RAPIDO, and by the national project COMBINE (with the contribution of the Italian Ministry of Foreign Affairs, Directorate General for the Country Promotion).

\section{References}

[1] J. Capmany, et al., "Microwave Photonics combines two worlds", Nature Photonics, Vol 1, June 2007.

[2] www.phodir.eu

[3] P. Ghelfi, et al., “A fully photonics-based coherent radar system”, Nature 507, pp. 341-345, 2014.

[4] F. Scotti et al., "In-Field Experiments of the First Photonics-Based Software-Defined Coherent Radar", J. Lightwave Technol., v. 32, n. 20, pp. $3365-3372,2014$.

[5] F. Laghezza et al., "Field trial of the first Photonic-Based Radar for Maritime Border Security and Harbor Protection", IRS2014.

[6] http://www.gemrad.com/products/military/radar.php

[7] F. Scotti et al., "Flexible True-Time-Delay Beamforming in a Photonics-Based RF Broadband Signals Generator", ECOC 2013, Th.2.B.5.

[8] F. Scotti et al., "Photonics-assisted dual band coherent radar system", EuRAD09-01, EURAD, Rome, Italy, Oct. 2014.

[9] F. Laghezza et al., "Photonics-Assisted Multiband RF Transceiver for Wireless Communications", J. Lightwave Technol., v. 32, n. 16, pp. $2896-2904,2014$. 